\title{
DESCENT FOR DISCRETE (CO)FIBRATIONS
}

\author{
MANUELA SOBRAL
}

\begin{abstract}
We characterize the (effective) $\mathbb{E}$-descent morphisms in the category Cat of small categories, when $\mathbb{E}$ is the class of discrete fibrations or the one of discrete cofibrations, and prove that every effective global-descent morphism is an effective $\mathbb{E}$-descent morphism while its converse fails.
\end{abstract}

KEYWorDs: Descent data, (effective) descent morphisms, (co)fibrations, discrete (co)fibrations, lax epimorphisms.

AMS SubJect Classification (2000): 18A25, 18D30, 18A20, 18 D05.

\section{Introduction}

Let $\mathbb{E}$ be the class of discrete cofibrations in Cat and $P: E \rightarrow B$ a functor between small categories. Denoting by $\mathbb{E}(B)$ the category of discrete cofibrations over $B$ and by $P^{*}: \mathbb{E}(B) \rightarrow \mathbb{E}(E)$ the pullback functor along $P$, we have the diagram, commutative up to isomorphism,

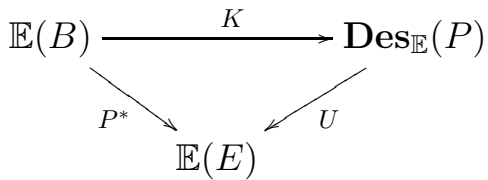

where $\operatorname{Des}_{\mathbb{E}}(P)$ is the category of $\mathbb{E}$-descent data for $P$, with $U$ and $K$ the forgetful and the comparison functor, respectively.

Now the question is to know if discrete cofibrations over the small category $E$ equipped with $\mathbb{E}$-descent data and the morphisms compatible with it essentially describe the category $\mathbb{E}(B)$, i.e. if $K$ is an equivalence. In this case we say that $P$ is an effective $\mathbb{E}$-descent morphism. When $K$ is full and faithful, i.e. $\mathbb{E}(B)$ is in one-to-one correspondence with its image by $K$, then $P$ is called an $\mathbb{E}$-descent morphism. We refer the reader to the papers [6] and [7] for a comprehensive introduction to descent theory.

Using the standard equivalence

$$
\mathbb{E}(B) \sim \operatorname{Set}^{B}
$$

The author gratefully acknowledges financial support from The Fields Institute, ATLANTIS 98-00-CAN-0017-00 and INTAS 97-31961. 
we describe a factorization of $P=\Phi \cdot \Psi$ and reduce the above question to the one of knowing when the functor $\mathbf{S e t}^{\Phi}$, which sends each functor $F: B \rightarrow$ Set to $F \cdot \Phi$, is an equivalence or full and faithful, respectively.

This gives a complete characterization of $\mathbb{E}$-descent morphisms and of effective $\mathbb{E}$-descent morphisms - see Theorem 1 and Theorem 2 with its corollaries, respectively.

We remark that

- dually, for the class $\mathbb{E}$ of discrete fibrations, we have $\mathbb{E}(B) \sim \operatorname{Set}^{B^{\text {op }}}$, and the same characterization theorems hold;

- Corollary 4 below naturally extends the similar result on preorders proved in [4].

Finally, we prove that the class of effective descent morphisms (i.e. of effective global-descent morphisms in the terminology of [6]) is strictly contained in the one of effective $\mathbb{E}$-descent morphisms.

\section{The factorization of $P$ through $Z(\mathbf{E q}(P))$}

For discrete cofibrations $\mathbb{E}(B) \sim$ Set $^{B}$ is an equivalence of Cat-indexed categories and, up to equivalence, we can identify $P^{*}: \mathbb{E}(B) \rightarrow \mathbb{E}(E)$ with the functor $\operatorname{Set}^{P}:$ Set $^{B} \rightarrow$ Set $^{E}$ which assigns to each functor $B \rightarrow$ Set its composite with $P$.

Accordingly, in diagram (1) the category $\operatorname{Des}_{\mathbb{E}}(P)$ is replaced by $\operatorname{Des}_{\Gamma}(P)$ for the Cat-indexed category

$$
\begin{aligned}
\Gamma: \mathbf{C a t}^{\mathrm{op}} & \longrightarrow \mathbf{C A T} \\
B & \longmapsto \text { Set }^{B} \\
(E \stackrel{P}{\rightarrow} B) & \longmapsto\left(\mathbf{S e t}^{P}: \mathbf{S e t}^{B} \rightarrow \mathbf{S e t}^{E}\right)
\end{aligned}
$$

where the natural isomorphisms $\Gamma(Q \cdot P) \cong \Gamma(P) \cdot \Gamma(Q)$ and $\Gamma\left(1_{B}\right) \cong 1_{\Gamma(B)}$ are equalities.

In Cat let $\mathbf{E q}(P)$ be the internal category

$$
E \times{ }_{B} E \times{ }_{B} E \underset{\pi_{12}}{\stackrel{\pi_{23}}{\rightleftharpoons}} E \times{ }_{B} E \underset{\pi_{1}}{\stackrel{\pi_{2}}{\rightleftarrows}} E
$$

induced by the kernel pair of $P$. By $E_{0}, E_{1}$ we denote the discrete categories of the objects and of the morphisms of the category $E$, respectively, and the same for $B_{0}$ and $B_{1}$ with respect to the category $B$. 
The category $\operatorname{Des}_{\Gamma}(P)$ can be described as the category of all pairs $(X, \xi)$ such that $X: E \rightarrow$ Set is a functor and $\xi=\left(\xi_{e, e^{\prime}}\right)$ is a family of maps $\xi_{e, e^{\prime}}: X(e) \rightarrow X\left(e^{\prime}\right)$ defined for $\left(e, e^{\prime}\right) \in E_{0} \times_{B_{0}} E_{0}$ and such that

$$
\xi_{e, e}=1_{X(e)} \text { and } \xi_{e^{\prime}, e^{\prime \prime}} \cdot \xi_{e, e^{\prime}}=\xi_{e, e^{\prime \prime}}
$$

for all $e, e^{\prime}$ and $e^{\prime \prime}$ with $P(e)=P\left(e^{\prime}\right)=P\left(e^{\prime \prime}\right)$ and the diagram

$$
\begin{gathered}
X(e) \stackrel{\xi_{e, e^{\prime}}}{\longrightarrow} X\left(e^{\prime}\right) \\
X(f) \downarrow \\
X\left(e_{1}\right) \underset{\xi_{e_{1}, e_{1}^{\prime}}^{\longrightarrow}}{\longrightarrow} X\left(e_{1}^{\prime}\right)
\end{gathered}
$$

commutes for all $(f, g) \in E_{1} \times_{B_{1}} E_{1}$. (See [3] for such a description when $\mathbb{E}$ is the class of étale morphisms in the category of finite topological spaces.)

Therefore, $(X, \xi)$ is a double functor from the double category $\mathbf{E q}(P)$ to the double category $S($ Set $)$ of commutative squares in Set. Indeed, it sends

- the horizontal arrows $e \rightarrow e^{\prime}$, i.e. the elements of $E_{0} \times_{B_{0}} E_{0}$ which we will denote by $\left[e, e^{\prime}\right]$, to the maps $\xi_{e, e^{\prime}}: X(e) \rightarrow X\left(e^{\prime}\right)$;

- the vertical arrows, i.e. the morphisms of $E$,

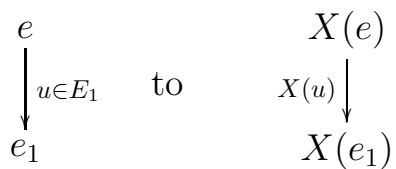

- and the squares

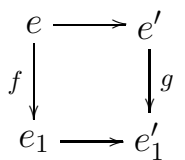

to the commutative squares

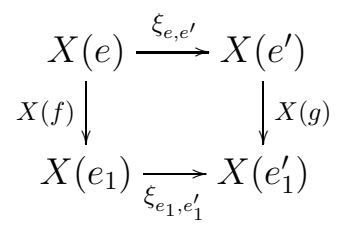


preserving horizontal and vertical composition as well as all types of identities.

The functor $S: \mathbf{C A T} \rightarrow$ DoubleCAT, which sends each category $\mathbf{C}$ to the double category of commutative squares in $\mathbf{C}$, has a left adjoint $Z$ (see e.g. [4]). So, in particular, double functors from $\mathbf{E q}(P)$ to $S$ (Set) are the same as functors from $Z(\mathbf{E q}(P))$ to Set.

The category $Z(\mathbf{E q}(P))$ can be described as the quotient category $\bar{E} / \sim$, where $\bar{E}$ is the free category generated by the disjoint union $E_{1} \cup E_{0} \times_{B_{0}} E_{0}$ and $\sim$ is the smallest equivalence relation which contains

- all pairs $((a, b), a b)$ if $a, b \in E_{1}$ or $a, b \in E_{0} \times_{B_{0}} E_{0}$;

- and $\left(a^{\prime}\left[e_{1}, e_{1}^{\prime}\right],\left[e_{2}, e_{2}^{\prime}\right] a\right)$

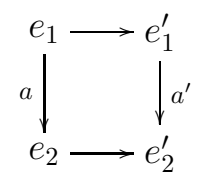

if $\left[e_{i}, e_{i}^{\prime}\right] \in E_{0} \times_{B_{0}} E_{0}$ and $P(a)=P\left(a^{\prime}\right)$.

That is, the objects in $Z(\mathbf{E q}(P))$ are the same as in $E$. A morphism in $Z(\mathbf{E q}(P))$ is the equivalence class of a morphism in $\bar{E}$ of the form
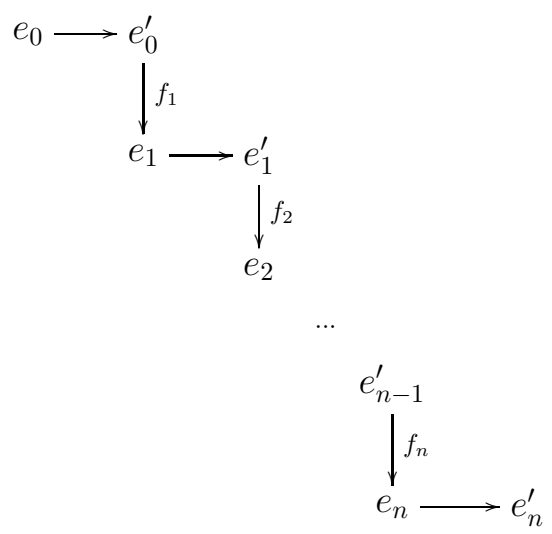

with $\left[e_{i}, e_{i}^{\prime}\right] \in E_{0} \times_{B_{0}} E_{0}$ and $f_{i} \in E_{1}$, which, like in [4], we will call a $n$-zigzag in $Z(\mathbf{E q}(P))$ from $e_{0}$ to $e_{n}^{\prime}$. 
The functor $P: E \rightarrow B$ has a factorization

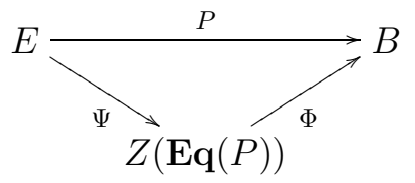

with $\Psi(f)=[f]$, the equivalence class of $f \in E_{1}$ in $\bar{E}, \Phi(e)=P(e)$ and $\Phi([z])$ is the composite $P\left(f_{n}\right) \cdot P\left(f_{n-1}\right) \cdots P\left(f_{2}\right) \cdot P\left(f_{1}\right): P\left(e_{0}\right) \rightarrow P\left(e_{n}^{\prime}\right)$ for the zig-zag $z$ given above.

\section{Characterization of (effective) $\mathbb{E}$-descent morphisms}

The diagram (1) can be identified, up to equivalence, with

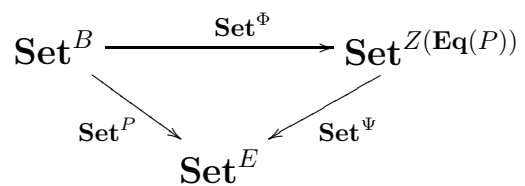

if $\mathbb{E}$ is the class of discrete cofibrations and with

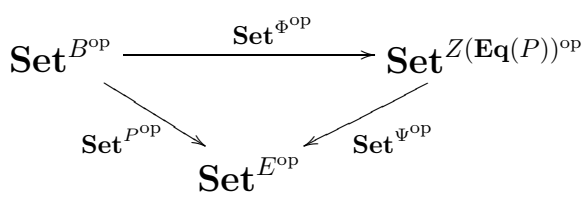

when $\mathbb{E}$ is the class of discrete fibrations.

Then $P$ is an (effective) $\mathbb{E}$-descent morphism for the class of discrete cofibrations if and only if $\mathbf{S e t}^{\Phi}$ is full and faithful (an equivalence, respectively). A similar result holds for (effective) descent morphisms relative to discrete fibrations and associated functors Set $^{\Phi^{\mathrm{op}}}$.

A functor $\Phi: A \rightarrow B$ in Cat is called a lax epimorphism if the functor $C^{\Phi}: C^{B} \rightarrow C^{A}$ is full and faithful, for every small category $\mathbf{C}$ (see [1] and references there).

A category $C$ is called connected if it is non-empty and there exists a finite sequence of $C$-morphisms, in either directions, between each pair of its objects.

For a morphism $h: b \rightarrow b^{\prime}$ in a small category $B$ we consider the category $h / / \Phi$ with objects the triples $(a, m, q)$ where $a$ is an object of $A$ and $m, q$ are 
morphisms in $B$ for which the diagram

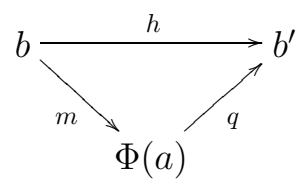

commutes.

The morphisms of $h / / \Phi$ from $(a, m, q)$ to $\left(a^{\prime}, m^{\prime}, q^{\prime}\right)$ are the morphisms $u: a \rightarrow a^{\prime}$ of $A$ for which the diagram

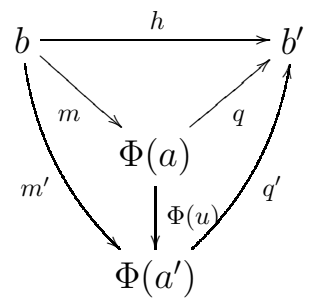

is commutative.

The following conditions are equivalent (cf. [1], Theorem 1.1):

(1) $\Phi$ is a lax epimorphism;

(2) Set $^{\Phi}$ is full and faithful;

(3) For every morphism $h: b \rightarrow b^{\prime}$ in $B$ the category $h / / \Phi$ is connected.

Theorem 1. The functor $P: E \rightarrow B$ is an $\mathbb{E}$-descent morphism, when $\mathbb{E}$ is the class of discrete fibrations or of discrete cofibrations, if and only $\Phi$ is a lax epimorphism.

Proof: Let $\mathbb{E}$ be the class of discrete cofibrations. Since $\mathrm{P}$ is an $\mathbb{E}$-descent morphism if and only if $\mathbf{S e t}^{\Phi}$ is full and faithful, by the equivalence (1) $\Leftrightarrow(2)$ we conclude that this means that $\Phi$ is a lax epimorphism.

But condition (3) is "self-dual". Hence, $\Phi: Z(\mathbf{E q}(P)) \rightarrow B$ is a lax epimorphism if and only if $\Phi^{\mathrm{op}}: Z(\mathbf{E q}(P))^{\mathrm{op}} \rightarrow B^{\mathrm{op}}$ is a lax epimorphism and the latter is equivalent to $P$ being a descent morphism with respect to discrete fibrations.

Theorem 2. The functor $P: E \rightarrow B$ is an effective $\mathbb{E}$-descent morphism, when $\mathbb{E}$ is the class of discrete fibrations or of discrete cofibrations, if and only if $\Phi$ is a full and faithful lax epimorphism. 
Proof: Let $\mathbb{E}$ be the class of discrete cofibrations. The functor Set ${ }^{\Phi}$ has a left adjoint $L$ (as well as a right adjoint) given by left Kan extension (right Kan extension, resp.) along $\Phi$.

The functor $P$ is $\mathbb{E}$-descent if and only the counit $\epsilon: L \cdot \operatorname{Set}^{\Phi} \rightarrow I d$ is a pointwise isomorphism. Furthermore, the fact that the unit $\eta: I d \rightarrow \operatorname{Set}^{\Phi} \cdot L$ is an isomorphism if and only if $\Phi: A=\operatorname{Set}^{Z(\mathbf{E q}(P)} \rightarrow B$ is full and faithful also follows from well-known facts:

- The unit $\eta$ is pointwise an isomorphism if and only if $\eta_{A(e,-)}$ is an isomorphism for all object $e$ of $A$, because each functor from $A$ to Set is a colimit of hom-functors and $\mathbf{S e t}^{\Phi} \cdot L$ preserves colimits;

- The left Kan extension of $A(e,-)$ along $\Phi$ is $B(\Phi(e),-)$;

- $\eta_{A(e,-)}: A(e,-) \rightarrow B(\Phi(e), \Phi-)$ is defined by $f \mapsto \Phi(f)$.

Now, since $\Phi^{\mathrm{op}}$ is a full and faithful lax epimorphism if and only if the same holds for $\Phi$, we conclude that the effective descent morphisms in Cat with respect to each one of these two classes of morphisms are the same.

From now on $\mathbb{E}$ will denote either of the classes considered in the previous theorems.

Corollary 3. The functor $P: E \rightarrow B$ is an effective $\mathbb{E}$-descent morphism if and only if

(i) For each morphism $h: P(e) \rightarrow P\left(e^{\prime}\right)$ in $B$ there exists a zigzag in $Z(\mathbf{E q}(P))$

$$
z=\left[e_{n}, e_{n}^{\prime}\right] f_{n} \cdots f_{2}\left[e_{1}, e_{1}^{\prime}\right] f_{1}\left[e_{0}, e_{0}^{\prime}\right],
$$

with $h=P\left(f_{n}\right) \cdots P\left(f_{2}\right) \cdot P\left(f_{1}\right)$, and such a zigzag is unique up to equivalence.

(ii) Every object $b \in B$ is a retract of an object in $P(E)$.

Proof: $(i) \Leftrightarrow \Phi$ is full and faithful.

If $\Phi$ is a lax epimorphism then $1_{b} / / \Phi$ is non-empty, for each $b \in B$, and so $b$ is a retract of $P(e)=\Phi(e)$ for an object $e$ in $E$.

Conversely, if $1_{b}=q \cdot m$ then, for each $h: b \rightarrow b^{\prime}, h / / \Phi$ is non-empty because $h=h q \cdot m$. Furthermore, for $\left(e^{\prime}, m^{\prime}, q^{\prime}\right) \in h / / \Phi$, the morphism $t=m^{\prime} \cdot q$ is the image of a zigzag $z$, unique up to the equivalence $\sim$,for 
which the diagram

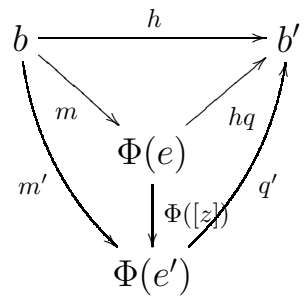

commutes.

The following corollary is a generalization of Theorem 1.2 in [4].

Corollary 4. If $B$ is a preordered set considered as a category, a functor $P$ : $E \rightarrow B$ is effective $\mathbb{E}$-descent if and only if the functor $\Phi: Z(\mathbf{E q}(P)) \rightarrow B$ is a category equivalence.

Proof: The non-trivial implication follows from the fact that if $b$ is a retract of $P(e)$ for $e \in E$ then $P$ and so $\Phi$ are essentially surjective on objects.

Examples 5. Every fibration, as well as every cofibration, $P: E \rightarrow B$ satisfying condition (ii) of Corollary 3 is an effective $\mathbb{E}$-descent morphism. If $P: E \rightarrow B$ is a fibration, $h: P(e) \rightarrow P\left(e^{\prime}\right)$ and $u: \bar{e} \rightarrow e^{\prime}$ is the cartesian lift for $h$ and $e^{\prime}$ then

$$
h=\Phi([u[e, \bar{e}]])=P(u)
$$

for a 1-zigzag $z=u[e, \bar{e}]$ in $Z(\mathbf{E q}(P))$. Since each n-zigzag is a composite of $n$ 1-zigzags, the proof that such a zigzag is unique, up to the equivalence $\sim$, follows from the following facts (which are essentially Proposition 2.1 and Corollary 2.2 in [4]):

- Two 1-zigzags in $Z(\mathbf{E q}(P))$ with the same image by $\Phi$ are equivalent: if $P\left(f_{1}\right)=P\left(f_{2}\right)$, for $f_{1}: e_{0}^{\prime} \rightarrow e_{1}, f_{2}: e_{0}^{\prime \prime} \rightarrow e_{1}^{\prime \prime}$ and $P\left(e_{i}\right)=P\left(e_{i}^{\prime}\right)=$ $P\left(e_{i}^{\prime \prime}\right)$ for $i=0,1$, then

$$
\left[e_{1}, e_{1}^{\prime}\right] f_{1}\left[e_{0}, e_{0}^{\prime}\right] \sim\left[e_{1}^{\prime \prime}, e_{1}^{\prime}\right]\left[e_{1}, e_{1}^{\prime \prime}\right] f_{1}\left[e_{0}, e_{0}^{\prime}\right] \sim\left[e_{1}^{\prime}, e_{1}^{\prime \prime}\right] f_{2}\left[e_{0}, e_{0}^{\prime \prime}\right] .
$$

- Every 2-zigzag is equivalent to a 1-zigzag:

$\left[e_{2}, e_{2}^{\prime}\right] f_{2}\left[e_{1}, e_{1}^{\prime}\right] f_{1}\left[e_{0}, e_{0}^{\prime}\right] \sim\left[e_{2}, e_{2}^{\prime}\right] f_{2} v\left[e_{0}^{\prime}, e_{0}^{\prime \prime}\right]\left[e_{0}, e_{0}^{\prime}\right] \sim\left[e_{2}, e_{2}^{\prime}\right] f_{2} v\left[e_{0}, e_{0}^{\prime \prime}\right]$

where $v$ is the cartesian lift for $P\left(f_{1}\right)$ and $e_{1}^{\prime}$. 
Fibrations which satisfy the prescribed condition are exactly those that are surjective on objects. In fact, for $b$ and $m: b \rightarrow P(e)$ in $B$, there is a cartesian lift $u: e^{\prime} \rightarrow e$ for $m$ and $e$, and so $b=P\left(e^{\prime}\right)$.

Hence, fibrations (and similarly cofibrations) are effective $\mathbb{E}$-descent morphisms if and only if they are surjective on objects.

\section{Effective descent versus effective $\mathbb{E}$-descent}

Under Beck-Chevalley condition effective descent reduces to monadicity, a result first published in [2]. In particular, $P$ is an effective descent morphism with respect to the basic Cat-indexed category if and only if $P^{*}$ : Cat $\downarrow$ $B \rightarrow$ Cat $\downarrow E$ is monadic.

The left adjoint to the restriction $P^{*} \mathbb{E}: \mathbb{E}(B) \rightarrow \mathbb{E}(E)$ of $P^{*}$ (denoted in diagram (1) also by $P^{*}$ ) exists but it is not defined by composition with $P$ on the left, like the left adjoint $P$ ! to $P^{*}$. In this case, the Eilenberg-Moore category with respect to the induced monad is not equivalent to the category $\operatorname{Des}_{\mathbb{E}}(P)$ of $\mathbb{E}$-descent data for $P$ (see Remark 7 ).

A functor $P$ is an effective descent morphism in Cat if and only if it is surjective on composable triples of morphisms. (See [5], Chapter VIII, Proposition 6.2).

From this characterization and Corollary 3 it follows that:

Theorem 6. Every effective descent morphism in Cat is an effective $\mathbb{E}$ descent morphism.

The converse is false: If $E$ and $B$ are the categories generated by the zigzag

$$
z=\left[e_{n}, e_{n}^{\prime}\right] f_{n} \cdots f_{2}\left[e_{1}, e_{1}^{\prime}\right] f_{1}\left[e_{0}, e_{0}^{\prime}\right] \quad \text { with } i \geq 3
$$

and by its image by $P$, respectively, then $P$ is an effective $\mathbb{E}$-descent morphism which is not surjective on composable triples.

It is easy to see that every (co)fibration which is surjective on objects is also surjective on composable triples and so that it is an effective descent morphism.

Remark 7. In our context, simple examples make clear that, in general, descent gives "more information" than monadicity. For instance, the functor $P: E=\{0,1\} \rightarrow B=\{0<1\}$ which sends $i$ to $i$, for $i=0,1$, is not even an $\mathbb{E}$-descent morphism. However both Set $^{P}$ and Set $^{P^{\text {op }}}$ are monadic. Indeed, Set $^{P}$ is monadic if and only if it is faithful and this is equivalent to $h / / P$ non-empty for all morphism $h$ of $B$ (cf. [1], Proposition 4.1). 


\section{References}

[1] J. Adámek, R. el Bashir, M. Sobral and J. Velebil, On functors which are lax epimorphisms, Theory Appl. Categ., Vol. 8, No. 20 (2001), 509-521.

[2] J. Bénabou and J. Roubaud, Monades et descente, Comptes Rendus Acad. Sc. Paris270 A (1970), 96-98.

[3] G. Janelidze and M. Sobral, Finite preorders and topological descent I, J. Pure Appl. Algebra 175 (2002), 187-205.

[4] G. Janelidze and M. Sobral, Finite preorders and topological descent II: étale descent, J. Pure Appl. Algebra 174 (2002), 303-309.

5] G. Janelidze, M. Sobral and W. Tholen, Beyond Barr exactness: effective descent morphisms, in: Categorical Foundations. Special Topics in Order, Topology, Algebra and Sheaf Theory, Cambridge Univ. Press (to appear).

6] G. Janelidze and W. Tholen, Facets of descent I, Appl. Categ. Struct. 2 (1994), 245-281.

[7] G. Janelidze and W. Tholen, Facets of descent II, Appl. Categ. Struct. 5 (1997), 229-248.

Manuela Sobral

Dep. Matemática, Univ. Coimbra, Apartado 3008, 3001-454 Coimbra, Portugal 